\title{
Analytical description of a trapped semi-ideal Bose gas at finite temperature
}

\author{
M. Naraschewski \\ Jefferson Laboratory, Department of Physics, Harvard University, Cambridge, MA 02138, USA \\ ITAMP, Harvard-Smithsonian Center for Astrophysics, 60 Garden Street, Cambridge, MA 02138, USA \\ D.M. Stamper-Kurn \\ Department of Physics and Research Laboratory of Electronics, Massachusetts Institute of Technology, Cambridge, MA 02139
}

(17 March 1998)

\begin{abstract}
Present experiments with Bose condensed gases can be largely described by a semi-ideal two-gas model. In this model, the condensate is influenced only by the mean-field repulsion among condensed atoms, while the thermal cloud is considered an ideal gas confined by an effective potential that consists of the external trap and the mean-field repulsion by the condensate. This simple, intuitive model provides explicit analytical expressions for the density distributions of the condensate and the thermal component. It describes the reduction of the condensate fraction relative to that of an ideal gas as a consequence of the positive chemical potential due to interactions in the condensate.
\end{abstract}

03.75.Fi,05.30.Jp

Recent experiments with Bose-Einstein condensed alkali gases (cf. e.g. [1,2]) have been successfully described by different approximation schemes. The diluteness of these gases, expressed as $n a^{3} \ll 1$ where $a$ is the twobody scattering length, allows a description in terms of an effective mean-field theory. At zero temperature the condensate can be described by the Gross-Pitaevskii equation [3, , 1 , a nonlinear Schrödinger equation. In present experiments interactions are strong enough so that the chemical potential $\mu$ is much larger than the level spacings $\hbar \omega_{x}, \hbar \omega_{y}$, and $\hbar \omega_{z}$ of the external harmonic potential. Therefore the use of the Thomas-Fermi approximation [0.6], which neglects the kinetic energy of the condensate, has lead to a tremendous simplification in the understanding of these experiments.

Recently, much theoretical and experimental attention has been focused on finite temperature properties of Bose-condensed gases. A semiclassical Hartree-FockPopov description has produced excellent agreement with experimentally measured equilibrium quantities [7]. Further, unlike in a spatially homogeneous gas, it was found that collective elementary excitations contribute very little to thermodynamic properties [7] due to the relatively small spatial overlap between condensate and thermal component in a harmonic trap. Thus, even a semiclassical Hartree-Fock approximation is a fairly accurate description of these gases, except for a small temperature range close to the critical temperature. For example, excellent agreement has been achieved by a comparison of Hartree-Fock density profiles with the results of a comprehensive Path Integral Monte Carlo calculation [8].

In this paper we introduce a further simplification of the self-consistent Hartree-Fock model by neglecting atomic interactions in the thermal component of the gas. Like the introduction of the Thomas-Fermi approximation [5] the reduction to Eqs. (4)-(6) and (12) has the important advantage of providing an explicit analytical description of a trapped Bose gas at finite temperature.
We consider atoms that are trapped in a generally anisotropic harmonic potential. Under the appropriate rescaling of energy and lengths, the harmonic potential has the form $V(\mathbf{r})=r^{2} / 2$. Here, the dimensionless cartesian coordinates of $\mathbf{r}$ are given in units of $\sqrt{\hbar \omega / m \omega_{x, y, z}^{2}}$, with $\omega=\left(\omega_{x} \omega_{y} \omega_{z}\right)^{1 / 3}$. Other lengths, like the thermal wavelength $\lambda_{T}$ and the scattering length $a$, are scaled in units of the natural length $\sqrt{\hbar / m \omega}$ of a harmonic oscillator with angular frequency $\omega$. The energy $V(\mathbf{r})$ as well as other energies are implicitly given in units of $\hbar \omega$.

Then, in the self-consistent Hartree-Fock model, the densities of the condensate and the thermal component are given as solutions of the two coupled equations [5:7]

$$
\begin{aligned}
& n_{0}(\mathbf{r})=\frac{\mu-r^{2} / 2-2 U n_{T}(\mathbf{r})}{U} \theta\left(\mu-r^{2} / 2-2 U n_{T}(r)\right) \\
& n_{T}(\mathbf{r})=\frac{1}{\lambda_{T}^{3}} g_{3 / 2}\left(e^{-\left(r^{2} / 2+2 U\left[n_{0}(\mathbf{r})+n_{T}(\mathbf{r})\right]-\mu\right) / k_{B} T}\right) .
\end{aligned}
$$

Here, the Thomas-Fermi approximation has been applied which neglects the kinetic energy of the condensate and implicitly finite size effects. The strength of the atomic interactions is given by $U=4 \pi a$. We use the thermal wavelength $\lambda_{T}=\sqrt{2 \pi / k_{B} T}$ and the Bose function $g_{3 / 2}$ is defined by $g_{\alpha}(z)=\sum_{j=1}^{\infty} z^{j} / j^{\alpha}$. The chemical potential $\mu$ is determined by the constraint of a given total atom number

$$
N=\int d \mathbf{r}\left[n_{0}(\mathbf{r})+n_{T}(\mathbf{r})\right] .
$$

For further reference, Eqs. (11) - (3) will be denoted as the interacting model. Even though it represents already a relatively concise description, these equations still have to be solved self-consistently. This is done numerically by an iterative procedure [7].

If one further neglects the mean-field repulsion from non-condensed atoms Eqs. (11) and (2) are solved by the explicit relations 


$$
\begin{aligned}
& n_{0}(\mathbf{r})=\frac{\mu-r^{2} / 2}{U} \theta\left(\mu-r^{2} / 2\right) \\
& n_{T}(\mathbf{r})=\frac{1}{\lambda_{T}^{3}} g_{3 / 2}\left(e^{-\left|r^{2} / 2-\mu\right| / k_{B} T}\right),
\end{aligned}
$$

with the chemical potential $\mu$ given by

$$
\mu=\frac{1}{2}(15 N a)^{2 / 5}\left(\frac{N_{0}}{N}\right)^{2 / 5} .
$$

These relations summarize a simple model of the partly condensed gas which we denote as the semi-ideal model. The condensate density $n_{0}$ is that of a zero temperature condensate with $N_{0}$ atoms, while the density $n_{T}$ of the non-condensed cloud is that of an ideal gas of bosons confined in the combination of the external potential and the repulsive mean-field potential due to the condensate atoms. Its wide range of validity has been confirmed by a numerical analysis in Ref. [9]. However, in order to make it an intuitively appealing, usable description of a trapped Bose gas, the condensate fraction has to be determined analytically as a function of temperature.

For later convenience let us introduce the reduced chemical potential $\bar{\mu}$

$$
\bar{\mu}=\frac{\mu}{k_{B} T}=\eta\left(\frac{N_{0}}{N}\right)^{2 / 5}\left(\frac{T}{T_{c}}\right)^{-1} .
$$

Here, the critical temperature $T_{c}$ is that of a trapped ideal gas [10,11 $k_{B} T_{c}=[N / \zeta(3)]^{1 / 3}$ where $g_{\alpha}(1)=\zeta(\alpha)$ was expressed in terms of the Riemann Zeta function. The reduced chemical potential depends on the scaling parameter $\eta[7$

$$
\eta=\frac{\mu_{T=0}}{k_{B} T_{c}}=\frac{1}{2} \zeta(3)^{1 / 3}\left(15 N^{1 / 6} a\right)^{2 / 5}
$$

which describes the strength of the atomic interactions within the condensate. The scaling parameter is independent of the system size when the thermodynamic limit is taken in the usual way $\left(N \rightarrow \infty, \omega \rightarrow 0, N \omega^{3}=\right.$ const. $)$. Due to its relatively weak $N^{1 / 6}$ dependence, $\eta$ assumes a value close to 0.3 in most recent experiments [7].

The condensate fraction is determined by integration over the thermal density distribution. For $T<T_{c}$, the result can be written in terms of incomplete Gamma functions 12

$$
\begin{aligned}
& \frac{N_{0}}{N}=-\frac{2}{\sqrt{\pi}} \frac{1}{\zeta(3)}\left(\frac{T}{T_{c}}\right)^{3} \\
& \times \times \sum_{j=1}^{\infty} \frac{1}{\left(j k_{B} T\right)^{3 / 2}}\left(\int_{0}^{\mu} d \epsilon \sqrt{\epsilon} e^{j(\epsilon-\mu) / k_{B} T}\right. \\
&\left.\quad+\int_{\mu}^{\infty} d \epsilon \sqrt{\epsilon} e^{-j(\epsilon-\mu) / k_{B} T}\right) \\
&=1-\frac{2}{\sqrt{\pi}} \frac{1}{\zeta(3)}\left(\frac{T}{T_{c}}\right)^{3} \sum_{j=1}^{\infty} \frac{1}{j^{3}}\left[e^{-j \bar{\mu}} \gamma(3 / 2,-j \bar{\mu})\right. \\
&\left.\quad+e^{j \bar{\mu}} \Gamma(3 / 2, j \bar{\mu})\right]
\end{aligned}
$$

Eq. (9) is still an implicit expression since it depends again on the reduced chemical potential. Therefore, $\bar{\mu}$ and $N_{0} / N$ are given as simultaneous solutions of Eqs. (7) and (9). For temperatures $T>T_{c}$ a condensate does not exist and the reduced chemical potential $\bar{\mu}<0$ is trivially defined by the single equation $g_{3}\left(e^{\bar{\mu}}\right)=\zeta(3)\left(T / T_{c}\right)^{-3}$.

Let us consider the results of the semi-ideal model in the limits of small and large $\bar{\mu}$, corresponding to $T \approx T_{c}$ and $T \approx 0$. Eq. (9) is therefore rewritten

$$
\frac{N_{0}}{N}=1-\frac{2}{\sqrt{\pi}} \frac{1}{\zeta(3)}\left(\frac{T}{T_{c}}\right)^{3} \sum_{j=1}^{\infty} \frac{1}{j^{3}} f(j \bar{\mu})
$$

in terms of the function

$$
f(x)=\int_{0}^{x} d \bar{\epsilon} \sqrt{\bar{\epsilon}} e^{\bar{\epsilon}-x}+\int_{x}^{\infty} d \bar{\epsilon} \sqrt{\bar{\epsilon}} e^{-(\bar{\epsilon}-x)},
$$

where the reduced energy $\bar{\epsilon}=\epsilon / k_{B} T$ has been introduced. The function $f(j \bar{\mu})$ can be expressed as a power series in $\sqrt{j \bar{\mu}}$, where $\bar{\mu}$ runs from $0\left(T=T_{c}\right)$ to $\infty$ $(T=0)$. Truncating the series after the lowest nontrivial order yields

$$
\begin{aligned}
\frac{N_{0}}{N} & =1-\left(\frac{T}{T_{c}}\right)^{3}\left[1+\frac{\zeta(2)}{\zeta(3)} \bar{\mu}\right] \\
& =1-\left(\frac{T}{T_{c}}\right)^{3}-\eta \frac{\zeta(2)}{\zeta(3)}\left(\frac{T}{T_{c}}\right)^{2}\left(\frac{N_{0}}{N}\right)^{2 / 5}
\end{aligned}
$$

The first two terms of Eq. (12) correspond to the condensate fraction of an ideal gas, whereas the third describes the influence of the condensate repulsion to lowest order in $\bar{\mu}$. This expansion adequately describes the solution of Eq. (9) over the entire range of temperatures, provided that $\eta \ll 1$. A further simplification is derived by solving Eq. (12) to lowest order in $\eta$, arriving at

$$
\frac{N_{0}}{N}=1-\left(\frac{T}{T_{c}}\right)^{3}-\eta \frac{\zeta(2)}{\zeta(3)}\left(\frac{T}{T_{c}}\right)^{2}\left[1-\left(\frac{T}{T_{c}}\right)^{3}\right]^{2 / 5}
$$

Within Eqs. 12 and (13), the only effect of the condensate interactions is to shift the chemical potential to positive values, raising the bottom of the effective potential which the thermal atoms experience. The effect of changing the shape of the potential enters only at higher orders of the expansion. Eq. (12) is thus a good approximation of Eq. (9) if most of the thermal atoms are spatially separated from the condensate, a condition satisfied for temperatures close to $T_{c}$ and for small $\eta$.

Eq. 12 is not expected to be a good description for extremely low temperatures. The understanding of this limit is facilitated by $f(x)$ obeying the differential equation

$$
\frac{d^{2}}{d x^{2}} f=f-2 \sqrt{x}
$$


The function $f(x)$ has to grow slower than $x^{3 / 2}$ for large values of $x$. Otherwise, the power series in Eq. (10) would not converge. As a consequence, the second derivative in Eq. (14) vanishes for large $x$. Thus, the asymptotic limit of the condensate fraction for low temperatures is given by $f(x)=2 \sqrt{x}$ or

$$
\frac{N_{0}}{N}=1-\sqrt{\eta} \frac{4}{\sqrt{\pi}} \frac{\zeta(5 / 2)}{\zeta(3)}\left(\frac{T}{T_{c}}\right)^{5 / 2} .
$$

However, it has been shown [7] that the leading term of the uncondensed fraction around zero temperature scales like

$$
\frac{N_{0}}{N}=1-\eta \frac{\pi^{2}}{2 \sqrt{2} \zeta(3)}\left(\frac{T}{T_{c}}\right)^{2}
$$

due to quasi-particle contributions which are included in neither the semi-ideal nor the interacting Hartree-Fock treatment.

According to Fig. 1, where $\eta=0.31$ has been assumed, the high temperature expansions Eqs. (12) and to some extent Eq. (13) are very good approximations of the exact condensate fraction Eq. (9) of the semi-ideal model over the whole range of temperatures $T<T_{c}$. In contrast, we have found that the validity of the low temperature limits of Eqs. (15) and (16) are restricted to a very small range around zero temperature which makes them nearly irrelevant for practical purposes.

The condensate fraction given by the approximate solution of the semi-ideal model in Eq. (12) is compared in Fig. 2 with the result of an iterative solution of the interacting model (Eqs. (1)-(3)). The difference between the curves is small except near the critical temperature, where the interactions among thermal atoms shift the critical temperature [13]. This shift is not exhibited by the semi-ideal model. The wide range of validity of the semi-ideal model has also been confirmed by a numerical comparison with a Hartree-Fock-Popov calculation [9]. The latter includes collective excitations, which are ignored in the models shown in Fig. 2. These lead to an additional but negligible decrease of the condensate fraction [7].

A direct comparison between the density distributions of the different models is given in Fig. 3 for $T / T_{c}=0.5$. Here, the Thomas-Fermi solution of the condensate density of Eq. (1) has been replaced by a solution of the full Gross-Pitaevskii equation. The main implication of the included kinetic energy of the condensate is a smoothed out condensate surface. Within the Thomas-Fermi approximation almost no difference is seen between the two curves. Even though the unaccounted existence of collective excitations changes the densities of condensate and thermal component close to the center of the trap by a significant amount, their influence on the total density is negligibly small for most temperatures [8].

In recent experiments, trapped Bose gases have been probed in-situ using either non-destructive dispersive imaging techniques 14], or off-resonant absorption imaging [15. These methods are important new tools in the understanding of trapped Bose gases, with distinct advantages over the previously used time of flight measurements of expanding atom clouds [1,2]. We therefore expect that the results of this paper will become useful for future in-situ experiments with trapped Bose gases. These new optical detection techniques measure column or line density profiles; we therefore determine these profiles from Eqs. (4) and (5) by integration over one or two dimensions.

In cylindrical coordinates, where the probe beam is directed along the $z$-axis, the according column densities are given by

$$
\begin{aligned}
& n_{0}(\rho)=\frac{4 \sqrt{2}}{3} \frac{\left(\mu-\rho^{2} / 2\right)^{3 / 2}}{U} \theta\left(\mu-\rho^{2} / 2\right) \\
& n_{T}(\rho)=\frac{2 \pi}{\lambda_{T}^{4}} \begin{cases}g_{2}\left(e^{\bar{\mu}(\rho)}\right) & \bar{\mu}(\rho)<0 \\
\int_{0}^{\infty} d \bar{\epsilon} \frac{1}{\sqrt{\pi \bar{\epsilon}}} g_{3 / 2}\left(e^{-|\bar{\epsilon}-\bar{\mu}(\rho)|}\right) & \bar{\mu}(\rho)>0\end{cases}
\end{aligned}
$$

where $\bar{\mu}(\rho)=\left(\mu-\rho^{2} / 2\right) / k_{B} T$. Equivalently, we obtain the line densities

$$
\begin{aligned}
n_{0}(z) & =\pi \frac{\left(\mu-z^{2} / 2\right)^{2}}{U} \theta\left(\mu-z^{2} / 2\right) \\
n_{T}(z) & =\frac{(2 \pi)^{2}}{\lambda_{T}^{5}} \begin{cases}g_{5 / 2}\left(e^{\bar{\mu}(z)}\right) & \bar{\mu}(z)<0 \\
2 \zeta(5 / 2)-g_{5 / 2}\left(e^{-\bar{\mu}(z)}\right) & \bar{\mu}(z)>0\end{cases}
\end{aligned}
$$

with $\bar{\mu}(z)=\left(\mu-z^{2} / 2\right) / k_{B} T$.

We therefore conclude that the proposed semi-ideal model of Eqs. (4)-(16) and (12) represents a rather good description of a trapped Bose gas at finite temperature provided that $\eta$ is not larger than the values $\eta \approx 0.3$ to 0.4 achieved in recent experiments. Its mathematical simplicity gives a much clearer conceptual picture than the self-consistent approaches that have been used before. The observed agreement of the semi-ideal model with a self-consistent Hartree-Fock calculation has confirmed that the density of the thermal component is too low to necessitate an interacting gas description. Instead, the observed depletion of the condensate fraction arises from condensate interactions, leading to a positive value of the chemical potential or equivalently to a raised bottom of the effective potential in which the thermal component resides. It is well described by Eq. (12), which is a low order expansion in $\bar{\mu}$. In contrast, the changed shape of the effective potential does not influence the thermal population in a noticeable way.

This work was supported by the Deutsche Forschungsgemeinschaft (DFG) and also the Office of Naval Research, the Joint Services Electronics Program (ARO) and the JSEP Graduate Fellowship Program. It is a pleasure to thank M. Olshanii, H.-J. Miesner, and W. Ketterle for many valuable discussions. After the completion of this work, E. Mueller brought to our attention a numerical calculation of the condensate fraction within the semi-ideal model [16]. 
[1] M. H. Anderson et al., Science 269, 198 (1995).

[2] K. B. Davis et al., Physical Review Letters 75, 3969 (1995).

[3] E. P. Gross, Nuovo Cimento 20, 454 (1961).

[4] L. P. Pitaevskii, Sov. Phys. JETP 13, 451 (1961).

[5] V. V. Goldman, I. F. Silvera, and A. J. Leggett, Physical Review B 24, 2870 (1981).

[6] G. Baym and C. J. Pethick, Physical Review Letters 76, 6 (1996).

[7] S. Giorgini, L. P. Pitaevskii, and S. Stringari, Journal of Low Temperature Physics 109, 309 (1997).

[8] M. Holzmann, W. Krauth, and M. Naraschewski, (unpublished).

[9] R. J. Dodd, K. Burnett, M. Edwards, and C. W. Clark, (unpublished).

[10] V. Bagnato, D. E. Pritchard, and D. Kleppner, Physical Review A 35, 4354 (1987).

[11] W. Ketterle and N. J. van Druten, Physical Review A 54, 656 (1996).

[12] I. S. Gradshteyn and I. M. Ryzhik, Table of Integrals, Series, and Products, 4 ed. (Academic Press, London, 1980).

[13] S. Giorgini, L. P. Pitaevskii, and S. Stringari, Physical Review A 54, R4633 (1996).

[14] M. R. Andrews et al., Science 273, 84 (1996).

[15] L. V. Hau et al., (unpublished).

[16] A. Minguzzi, S. Conti, and M. P. Tosi, Journal of Physics: Condensed Matter 9, L33 (1997).

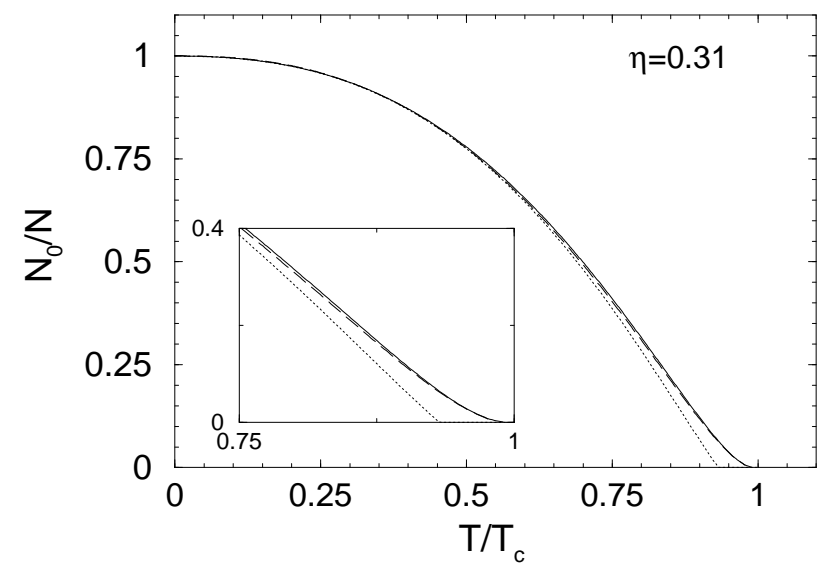

FIG. 1. Condensate fraction as a function of temperature, given by the semi-ideal model. The dashed line shows the exact solution Eq. (9) of the semi-ideal model. The solid line represents its high-temperature limit Eq. (12). The explicit expression of Eq. (13) is given by the dotted line. This plot demonstrates the validity of Eq. (12) over the whole range of temperatures $T<T_{c}$. The explicit approximation of Eq. (13) fails only in the vicinity of the critical temperature (shown in the inset).

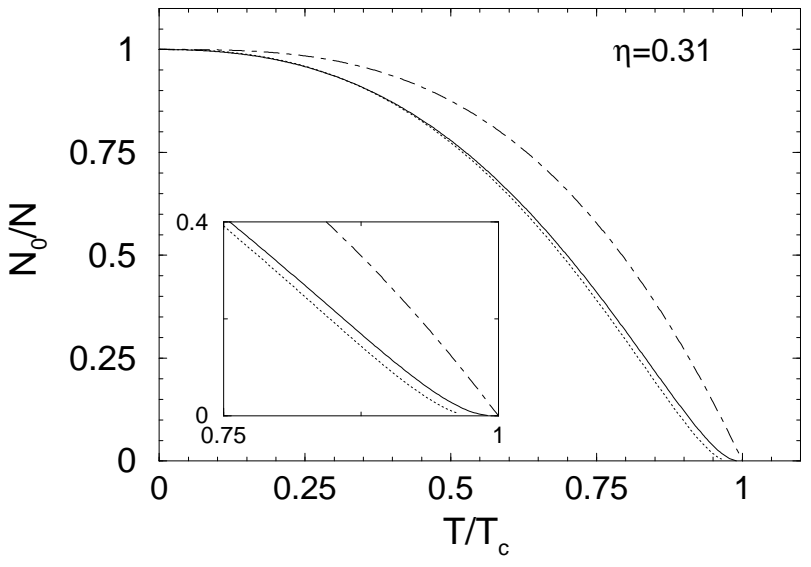

FIG. 2. Condensate fraction plotted as a function of temperature, comparing the discussed models. The ideal gas condensate fraction is given by the dashed-dotted line. The dotted line is a numerical solution of the Hartree-Fock Eqs. (11)-(3). The solid line is obtained from the analytical approximation Eq. (12), corresponding to the solid line of Fig. 1. Except near the critical temperature (shown in the inset), the semi-ideal model well approximates the solution of the interacting model. The critical temperature $T_{c}$ refers to the case of an ideal gas.

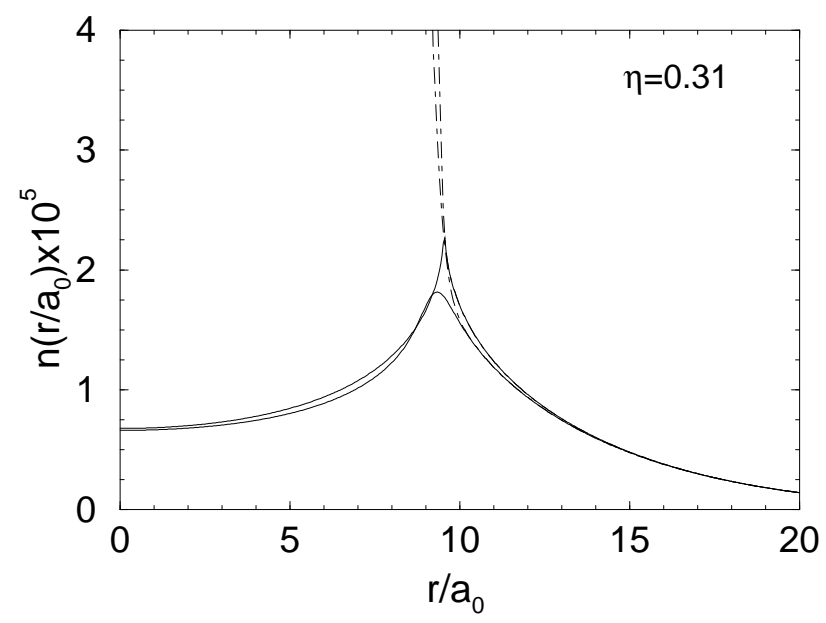

FIG. 3. A comparison of the density distributions at $T / T_{c}=0.5$ predicted by the two discussed models. The upper solid line shows the density of the thermal component as given by Eq. (1). In comparison, the lower solid line has been calculated numerically using Eq. (2). However, a numerical solution of the full Gross-Pitaevskii equation has been used instead of the Thomas-Fermi approximation of Eq. (1). A small difference exists at the edge of the condensate due to the neglect of the kinetic energy in the semi-ideal model. However, the total density is only weakly affected by this effect, as is seen by the two dashed-dotted lines. Again, the upper dashed-dotted line refers to the total density given by the semi-ideal model while the lower curve corresponds to the self-consistent Hartree-Fock approximation. Here, a total atom number of $N=5 \times 10^{6}$ was assumed. 